\title{
New Geometric Transform Based on Stochastic Geometry in the Context of Pattern Recognition
}

\author{
Nikolay Fedotov ${ }^{1}$, Lyudmila Shulga \\ ${ }^{1}$ Penza State University, 40, Krasnaya St., Penza, 440026, Russia \\ fedotov@diamond.stup.ac.ru, milena_s@penza.com.ru
}

\begin{abstract}
Application of stochastic geometry methods to pattern recognition is analyzed. The paper is based on Trace-transforms of original images introduced by [1] into images on the Möbius band. The ability of a Trace-transform to solve such structuralistic problems as segmentation, analysis of objects' relative position, and their number evaluation, is established. Feasibility of image nonlinear filtering through Trace-transforms is considered. Based on the new geometric transform, a new approach towards the construction of features, independent of images' motions or their linear transforms, is put forward. A prominent characteristic of the group of features under consideration is that we can represent each of them as a consecutive composition of three functionals.
\end{abstract}

\section{Introduction}

The article presents the basics of a new theory to construct recognition features, which are dependent on image motions and affine transforms.

The key element of the theory is a newly introduced geometric transform, which the authors chose to call a "Trace-transform". An outstanding characteristics of the group of new features obtained on the basis of the above transform, is its distinctive structure - a sequential composition of three functionals.

Such a structure of features helps automatically generate a greater number of new constructive sign of recognition. Such a powerful shift of stress from the decision procedures onto features' mass application, accounts for speaking about a new interpretation of images, and enhancing intellectual power of recognition systems.

\section{Trace-Transform}

Let $F$ denote a finite image. Given straight line $l, g$ characterizing the location of $l$ and the image as to each other, is to be computed according to a certain rule $\mathbf{T}$ : $g=\mathbf{T}(l, F)$; map $\mathbf{T}$ is called a functional. The property required for us here is the computational independence of an object motion. Therefore the only requirement 
imposed on $\mathbf{T}$ is stated as follows. Let the image have been shifted and rotated, and $F^{\prime}$ be the new one. For the same shift and rotation, $l$ will become a straight line $l^{\prime}$, thus remaining, "frozen" into the image. It is required that $\mathbf{T}(l, F)=\mathbf{T}\left(l^{\prime}, F^{\prime}\right)$. The equality is to hold for all straight lines and all acceptable images. We may call the property a complete invariance of functional $\mathbf{T}$. It should be noted that the concept of complete invariance extends pattern recognition capabilities substantially, for it is not necessarily be the number of intersections, intersection length, etc.

Functionals can be selected to describe finer properties of neighborhood, such as neighborhood morphology, or topological characteristics. For a full-color image of a variable brightness a great number of such functionals could be selected. Hence, the range of functionals and images to be processed widens considerably.

Just like in stochastic geometry, random value $g=\mathbf{T}(l, F)$ is defined, its distribution being independent of image shifts and rotations. Therefore, numeric characteristics of the random value may again serve as image features, which are to be established with the help of special engineering devices and systems. The limitation of the new family of features is that they originally lack an explicit geometrical meaning, and their differentiating capability is a priori unknown. However for pattern recognition, it proves not very important, experimental testing being decisive.

To understand that the generalization proposed in a certain aspect exhaust its own possibilities, we are going to state the theory of Trace-transform. Polar coordinates introduced to the plane, $l$ is characterized by distance $p$ from the origin to $l$, and by angle $\theta$ (up to $2 \pi$ ) of its directional vector: $l=\{(x, y): x \cos \theta+y \sin \theta=p\}$, $l=l(\theta, p)$, where $x, y$ are Cartesian coordinates on the plane. If we allow $p$ to take negative values, too, then $l(\theta, p)=l(\theta+\pi,-p)$.

Thus, a set of all directed straight lines intersecting a circle of radius $R$ with the center in the origin (the "retina"), is unambiguously parameterized by set $\Lambda=\{(\theta, p): 0 \leq \theta \leq \pi,-R \leq p \leq R\}$, which provided parameters $(0, p)$ and $(\pi,-p)$ define the same straight line. The set of straight lines on the retina are clearly seen to be topologically nothing but a Möbius band. Thus, the set of numbers $\mathbf{T}(l(\theta, p), F)$, depending on a point on Möbius band $\Lambda$, is a certain image transform, which we may call a Trace-transform. If, for instance, a matrix represents a Trace-transform in numerical analysis, we may call it a Trace-matrix. If axis $0 \theta$ is directed horizontally, and axis $0 p$ vertically, matrix element, indicated $(i, j)$, i.e. value $\mathbf{T}\left(l\left(\theta_{j}, p_{i}\right), F\right)$, is in point $\theta_{j}, p_{i} . \theta_{j}$ and $p_{i}$ are here certain values of uniform discrete grids on the axes mentioned. Along the horizontal axis, matrix is $2 \pi$-periodic, its columns rotating within each interval of length $\pi$.

In addition, let us consider, that if $l$ does not intersect the image, $\mathbf{T}(l, F)$ is a given number (say, 0 ), or another fixed element, if $\mathbf{T}$ is nonnumeric. In this case, a new image $\mathbf{T}(F)$ corresponds to the original image $F,(\mathbf{T}(l(\theta, p), F)$ may be treated as an image which characteristics at $(\theta, p)$ are its Trace-image. 
Fig. 1,a explains the computation of a Trace - transform. It shows how to obtain a binary function $f(\theta, p, t)$ of a real variable for a scanning line $l$.

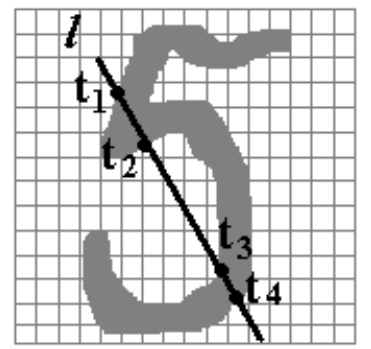

a)

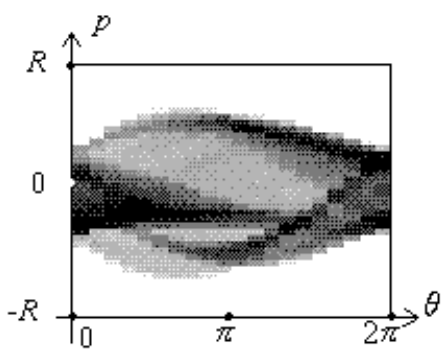

b)

Fig.1. Example of calculation a binary function $f(\theta, p, t)$ for given image a scanning line $l$ and corresponding Trace-Transform

Function $f(\theta, p, \bullet)$ equals 1 within the interval $\left(t_{1}, t_{2}\right)$ and $\left(t_{3}, t_{4}\right)$. Within other precise it equals 0 . Let $\mathbf{T}$ stand for a functional applied to the function, its independent variable being designated by $t$. Thus we get $g(\theta, p)=\mathbf{T} f(\theta, p, t)$. We call function $g$ result of Trace - transform.

For a two-level image, such a Trace-transform could be obtained in case T $f(\theta, p, \bullet)$ is the total of all the intervals from the domain of the function to be defined. For Fig. 1 ,a it is the value of $t_{2}-t_{1}$ and $t_{4}-t_{3}$ segments' total.

For instance, let $\mathbf{T} f(\theta, p, t)$ be maximum interval within function $f(\theta, p, \bullet)$ domain. In Fig. $1, \mathrm{a}$ it is the value of $t_{2}-t_{1}(\max G)$. If we determine a similar $\mathbf{T} f(\theta, p, \bullet)$ for an aggregate of scanning lines intersecting the image of a image in Fig. $1, \mathrm{a}$ at various angles $\theta$ and various distances $p$, we can get its Trace - image in Fig. 1,b.

Note that the famous Radon transform can be viewed as an example of a Trace transform. Brightness integral value summed over all scan lines for all directions, is Radon transform. In case $\mathrm{T} f_{p, \theta}=\int_{-\infty}^{\infty} f_{p, \theta}(t) d t$ - brightness integral value $f(p, \theta)$ along a scan line with parameters $(\theta, p)$, the collection $\{\mathrm{T} f p, \theta\}, p \in R, \theta \in[0,2 \pi]$ bears all information on the image.

In the context of two-level images, Radon transform will lie in summing up blackened elements along each scan line. Hence, choosing an appropriate functional, we can make Trace-transform realize Radon transform. This is a particular case, though. In other particular cases one can both make Trace -transform match other well-known geometric transforms — Fourier, Hough, etc. — or outstep [2]. 


\section{Property of Trace-transform}

Trace-transform proves convenient to study objects' movements and similarity transform within the retina.

Let us briefly consider how image $\mathbf{T}(F)$ changes after initial image $F$ has been shifted and rotated. If the original image rotates, its Trace-image shifts along the horizontal axis $0 \theta$. If the original image shifts to a certain vector, its Trace-image undergoes changes as follows. For convenience they are to be stated in terms of Trace-matrices. Columns remain unchanged and stay in their places, though may shift up or down. Shift vector specifies numbers $a$ and $b$ such that a column with coordinate $\theta_{i}$ shifts vertically to $a \cos \left(\theta_{i}-b\right)$. It should be noted that the description is to be totally rigorous if only we consider Trace-matrix continuous, i.e., $i$ and $j$ are continuous parameters. Fig. 1 shows the image of an original image and the result of its Trace- transformation, i.e. its Trace-transform.

Fig. 2,a and 2,b show the images after it has been shifted, and the corresponding Trace-transform. Fig. 2,d contains the image subject to scale transformations, and the corresponding extended Trace - transform. Fig. 2,c depicts the rotated image of the given image, and its Trace-transform.

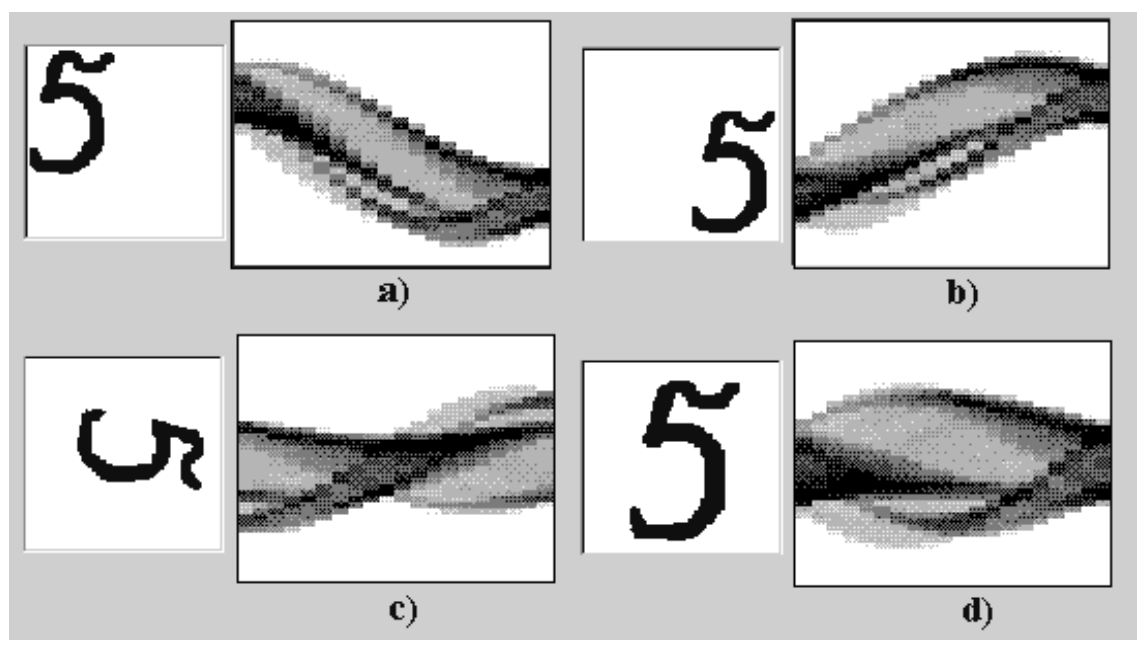

Fig.2. Image under transformations and corresponding Trace-transforms.

A common Euclidean measure $d \theta d p$ of the Möbius band is invariant to the transforms indicated, hence distribution density of every function (image functions $\mathbf{T}(F)$ in our case) defined on the Möbius band is independent of the transforms indicated, i.e. if image $F$ is shifted and rotated to $F^{\prime}$, then distributions of $\mathbf{T}(F)$ and $\mathbf{T}\left(F^{\prime}\right)$ image function values are identical. That is why their values may be treated as 
random functions independent of the original image motions. This proves that invariance holds for the above generalization of features.

Let us consider a valuable feature of Trace-transforms to tackle certain problems of image analysis normally solved structurally: through segmentation, or evaluation of objects' relative position and number. Fig. 3, a shows an object to be viewed as having a single-connected, and its Trace-matrix, Fig. 3,b and Fig. 3,c showing segmented images and their Trace-matrices.

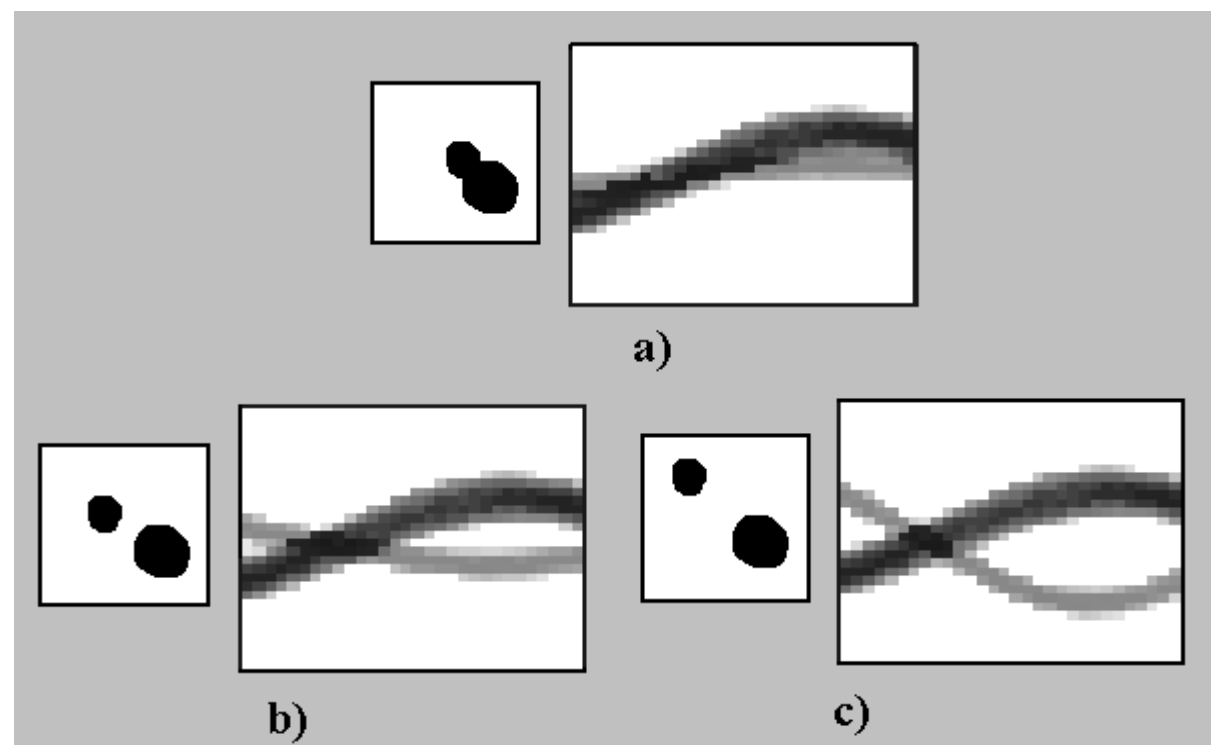

Fig.3. The experiment on a single-connected and doubly-connected images and corresponding Trace-matrixes

The example, which proves structuralistic features of Trace-transform, applies to the field of technical diagnostics. A problem of recognizing welded joints' pores was being solved based on the results of their radiographic inspection. Fig.3 shows one of the defections typical for welded joints - spherical pores. Instructions on radiographic inspection request that chains of pores be differentiated from single pores and other defections, such as slag inclusions, incomplete penetration, etc.

Moreover, this type of defection is suggested to be differentiated according to the relative position of pores within the chain, as well as to the number, and the size (the diameter) of pores.

Through a Trace-transform, one can implement image nonlinear filtration to reduce image noise and quantization. This valuable feature is pointed with an example from the field of technical diagnostics.

It has been already mentioned that a transitional image of an original obtained on the Mobius band, i.e. a Trace-transform, can be Trace-transformed again. A retransform has been performed to point the filtering abilities of a Trace-transform. Fig.4,a shows a Trace-transform of a welded joint with inclusions. For a $\mathbf{T}$-functional 
of the transform, the number of segments has been taken to be cut out by the image on the scanning line. The given Trace-matrix both contains information on the welded joint, its shape, and the existing pores (the high-leveled gray indicates the matrix elements corresponding to the welded joints' pores).

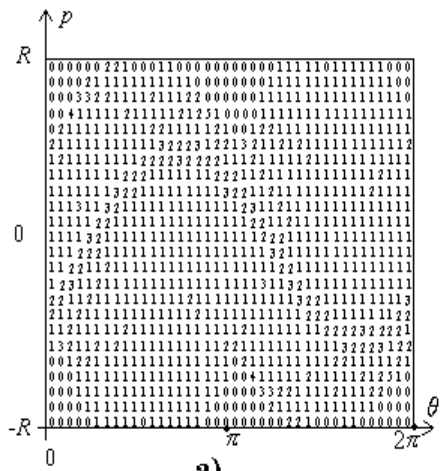

a)

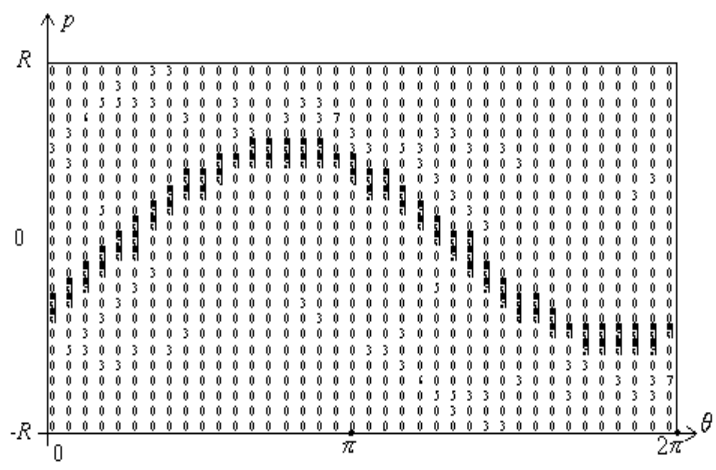

b)

Fig. 4. Nonlinear filtering by using Trace-transform

For getting information only about flaws of welded join we propose to use more completed T-functionals. For example, correspondent T-functional (1) let us to get information about flaw (Fig. 4,b) excepting welded join information:

$$
a_{i j}^{\prime}=\left\{\begin{array}{c}
0, \text { if } a_{i j}=0 \\
10 \lg a_{i j}, \text { if } a_{i j}>0
\end{array}\right.
$$

where $a_{i j}$ are number of intersections scanning line of given image

Through the Trace-transform, we get a matrix of an original dimension, which elements almost unexceptionally differ from zero if they correspond to a pore (Fig.4,b).

Other types of T-functional have been found to replace certain types of image preprocessing, such as inversion, and filling closures.

The most valuable feature of the Trace-transform is that it proves a source of a new class of recognition features which have a triple structure (triple features [3,7]). Such features are to be formed through the consecutive, column-wise compression of information contained in the Trace-matrix, with a Diametric functional $\mathrm{P}$. The operation results in a $2 \pi$-periodic function. With this, the next step is to obtain the image feature $\Pi(F)$ through a Circus functional $\Theta$ : $\Pi(F)=\Theta \circ \mathbf{P} \circ \mathbf{T}(F \circ L(\theta, p, t))$. See [3, 4] for more details.

It should be noted that one could enhance non-linear filtration applying the filtering capabilities of both the Trace-functional and other functional of triple structure. 


\section{Recognition systems}

Program systems of pattern recognition based on stochastic geometry methods, have been designed and constructed. Their basic structure elements are: a subsystem of automatic computer feature generation, a database of functional, a subsystem of feature selection, and a subsystem of decision procedure.

A subsystem of feature selection reduces their redundancy through sifting out noninformative features, and forms a minimum set of effective features. The research conducted to find out how to minimize the feature space, demonstrates that applying minimization procedure based on the decomposition of Karhunen-Loeve, implements the decision procedures that are the simplest, ergo the fastest to compute.

The system has been successfully tested to recognize biological microobjects boasting of complicated morphology.

It is to be emphasized that for certain recognition applications, the system based on stochastic geometry methods could be modified and simplified. For instance, to solve certain technical diagnostics problems, a system has been created to recognize defects in welded joints through the results of radiography control. The analysis demonstrates that the most efficient way is to filtering and binarization images on the basis of a relevantly small number of triple features. In such a case, a special procedure to minimize feature space comes unnecessary.

\section{Conclusion}

Properties of new geometric Trace-transform are presented which is generation other well-known geometric transforms - Fourier, Chough, Radon.

Trace-transform proves convenient to study objects' movements and similarity transform within the retina.

The ability of a Trace-transform to solve such structuralistic problems as segmentation, analysis of objects' relative position, and their number evaluation, is established. Feasibility of image nonlinear filtering through Trace-transforms is considered.

A feature structured as a consecutive composition of three functionals, helps construct a great number of features. For instance, we could get thousands of features for 10 variants of functionals. Moreover, they could be obtained in the mode of automatic computer generation automatically, (i.e.) in the mode of computer generation.

At present, over 200 functionals applicable for feature construction, have been identified in various fields of mathematics, probability theory, mathematical statistics, theory of series, and fractal theory. Through the application of the such features, one could enhance recognition validity and universality, for the functionals genetically pertain to the appropriate fields of mathematics.

Flexibility of a triple structure is an advantage of the suggested approach. One could automatically form triple features to recognize effectively objects of variable shapes, as well as features to find out how objects are positioned as to one another, 
and the number of the objects, and certain features to reflect objects' geometry. Computation in such cases could be performed in a similar manner, taking advantage of a parallel processing. That proves a high degree of the whole recognition algorithm universality.

\section{References}

1. Fedotov N.G., Metody Stokhasticheskoi Geometrii v Raspoznavanii Obrazov (Methods of Stochastic Geometry in Patters Recognition), Radio i Svjaz, Moscow (1990)

2. Fedotov N.G and Kadyrov A.A., Image Scanning in Machine Vision Leads to New Understanding of Image, Fifth Int Workshop on Digital Image Processing and Computer Graphics, Vol. 2363, Proc. SPIE (1994) 256-261

3. Kadyrov A.A and Fedotov N.G. Triple Features, Pattern Recognition and Image Analysis, Vol. 4 (1995)

4. Nikolay G. Fedotov, Lyudmila A. Shulga. New Ways to Form Features for Pattern Recognition on the Basis of Stochastic Geometry, $12^{\text {th }}$ Scandinavian Conference on Image Analysis, Bergen, Vol. I.II, Norway, (2001) 373-380

5. Fedotov N.G., Shulga L.A. New Theory of Pattern Recognition on the Basis of Stochastic Geometry, WSCG'2000 Conference Proceedings, ISBN 80-7082-6126, Vol. 1(2), (2000) 373-380

6. Nikolay G. Fedotov, Lyudmila A. Shulga. Enhancing Intellectual Power of Recognition Systems Based on New Pattern Recognition Theory, Proceedings 2002 IEEE International Conf. on Artificial Intelligence Systems (ICAIS 2002), IEEE Computer Society, Los Alamos, California, (2002) 192-197

7. Fedotov N.G., Nikiforova T.V. Technicekaya defecktospopiya na osnove novoi teorii raspoznavaniay obrazov (Technical defectoscopy based on the new theory of pattern recognition), Measurement Technique, Vol.12, (2002) 27-31 